\title{
Functional characterization of canine wild type glucocorticoid receptor and an insertional mutation in a dog
}

\author{
Kosei Yamanaka ${ }^{1}$, Masaru Okuda $^{2}$ and Takuya Mizuno ${ }^{1 *}$
}

\begin{abstract}
Background: Glucocorticoids, among the most widely utilized drugs in veterinary medicine, are employed to treat a wide variety of diseases; however, their use often induces adverse events in dogs. The efficacy of glucocorticoids usually depends on dosage, although differences in sensitivity to glucocorticoids in individual animals have been reported. Glucocorticoids bind to the cytoplasmic glucocorticoid receptor (GR), which is expressed in almost all cells. These receptors are key factors in determining individual sensitivity to glucocorticoids. This study examined individual differences in glucocorticoid sensitivity in dogs, focusing on reactivity of the GR to prednisolone.

Results: We first molecularly cloned the GR gene from a healthy dog. We discovered a mutant GR in a dog suspected to have iatrogenic Cushing syndrome. The mutant GR had extra nucleotides between exons 6 and 7, resulting in a truncated form of GR that was 98 amino acids shorter than the wild-type dog GR. The truncated GR exhibited very low reactivity to prednisolone, irrespective of concentration.

Conclusions: We have identified the truncated form of canine GR in a dog with iatrogenic Cushing syndrome. This truncated form showed the very less sensitivity to glucocorticoid in vitro, unfortunately, we could not elucidate its clinical significance. However, our data is a first report about the function of canine GR, and will facilitate the analysis of canine glucocorticoid sensitivity.
\end{abstract}

Keywords: Canine, latrogenic Cushing syndrome, Glucocorticoid receptor, Reporter gene assay

\section{Background}

Glucocorticoids are used to treat numerous diseases, inflammatory conditions, transplant rejection, and various autoimmune disorders in both human and veterinary medicine. The primary expected effects of glucocorticoids are anti-inflammatory and immunosuppressive in nature and depend on the dose of medication. Generally, whereas lower doses of glucocorticoids induce only antiinflammatory effects, higher doses are associated with additional immunosuppressive effects. However, even in the same species, individual differences in response to glucocorticoids can occur [1-3].

The functions of glucocorticoids are initiated upon binding to the cytoplasmic glucocorticoid receptor (GR),

\footnotetext{
* Correspondence: mizutaku@yamaguchi-u.ac.jp

${ }^{1}$ Laboratory of Molecular Diagnostics and Therapeutics, Joint Faculty of Veterinary Medicine, Yamaguchi University, 1677-1 Yoshida, Yamaguchi-shi, Yamaguchi 753-8515, Japan

Full list of author information is available at the end of the article
}

which is expressed in most types of cells. Because GRs are nuclear receptors, glucocorticoid/GR complexes are translocated to the nucleus, where they are involved in regulating the transcription of many genes. GRs have several important functional domains: an N-terminal regulatory domain (NTD), a DNA-binding domain (DBD), and a ligand-binding domain (LBD). Cloning and sequencing of guinea pig GR revealed 24 amino acid differences in the LBD compared to the human GR. Lower affinity of the guinea pig GR for dexamethasone in comparison with mouse and human GR was attributed to 4 of these 24 different amino acid residues [4]. In addition, guinea pigs have higher levels of circulating cortisol than mice and humans. Individual differences in sensitivity to glucocorticoids due to single nucleotide polymorphisms or GR structural abnormalities have been frequently reported in humans $[1-3,5]$.

Compared with humans, domesticated dogs and cats are relatively resistant to glucocorticoids [6]. However, 
the mechanism underlying this species-specific difference remains unclear, and its elucidation has awaited molecular characterization of the dog GR. In this study, we molecularly cloned the canine $G R$ from three healthy dogs and a dog undergoing veterinary treatment for suspected iatrogenic Cushing syndrome. We determined the sensitivity of each GR to prednisolone using a reporter gene assay. In addition, we identified structural defects in the GR of the dog undergoing veterinary treatment and determined the GR sensitivity in this dog.

\section{Results}

\section{Clinical description of a dog suspected of having iatrogenic Cushing syndrome}

A 6-year-old spayed, mixed-breed dog was referred to the Yamaguchi University Animal Medical Center for detailed examination of skin disease. The dog was tentatively diagnosed with pemphigus foliaceus at a private hospital 9 months previously. The dog had been treated with prednisolone $(0.7 \mathrm{mg} / \mathrm{kg} /$ day orally) for approximately 3 months. After the dog was started on the medication, polyuria, polydipsia, and abdominal distension were observed. The pemphigus foliaceus was neither ameliorated nor aggravated. After prednisolone withdrawal, calcinosis cutis was observed on the dorsal skin. Two months later, on the day of consultation at our hospital, the adverse reaction due to glucocorticoid therapy had already disappeared, except for extremely severe calcinosis cutis over the entire dorsal skin (Fig. 1a and b). Table 1 shows the results of a blood examination on this day.

\section{Nucleotide and deduced amino acid sequences of canine GR cDNA}

Before analyzing the nucleotide sequence of the dog's $G R$, the nucleotide sequence of the canine $G R$ was molecularly cloned and analyzed, as it had not been reported previously. PCR amplification of the dog GR cDNA (derived from one liver and two PBMCs all from healthy beagles) using the primers YTM673 and YTM674 provided a DNA fragment with an expected size of approximately $2500 \mathrm{bps}$.
Nucleotide sequencing of the full-length $\operatorname{dog} G R$ revealed a cDNA clone covering 2522 bps and containing an open reading frame of $2343 \mathrm{bps}$ encoding a protein of 780 amino acids (Fig. 2). The nucleotide sequence of the dog GR was 91, 87, and 89\% identical to that of human $G R \alpha$ (GenBank Accession No. P04150), mouse (Mus musculus) GR $\alpha$ (GenBank Accession No. P06537), and guinea pig (Cavia porcellus) GRa (GenBank Accession No. P49115), respectively.

Alignment of the predicted amino acid sequence of the $\operatorname{dog} G R$ cDNA clones with those of the human, mouse, and guinea pig cDNAs is shown in Fig. 3. The deduced amino acid sequence of the dog GR cDNA exhibited 92, 89 , and $88 \%$ homology with that of the human, mouse, and guinea pig GR $\alpha$ polypeptides, respectively. Consistent with the high homology of the predicted $\operatorname{dog} \mathrm{GR} \alpha$ amino acid sequence to those of other species, the $\operatorname{dog} G R \alpha$ appeared to be composed of an $\mathrm{N}$-terminal regulatory domain (NTD), a DNA-binding domain (DBD), a hinge region (HR), and a ligand-binding domain (LBD), similar to the human, mouse, and guinea pig receptors. These results suggest that the function of the dog GR $\alpha$ is similar to that of other species. Of the functional domains, the DBD was the most conserved between species, and no amino acid differences were found between the human, dog, and mouse GRos. The LBD also exhibited high homology between species, whereas the NTD exhibited less homology.

Phosphorylation of the human GR $\alpha$ at many sites (T8, S45, S113, S134, S141, S203, S211, S226, S234, S267, and S404) has been reported, and this is considered to play an important role in GR $\alpha$ functions, such as transactivation and nuclear export [7-9]. Compared with the human receptor, most of the phosphorylation sites were conserved in $\operatorname{dog}$ and mouse GR $\alpha$, except those at residues 8 and 234. The threonine at residue 8 was less conserved between species. The serine at residue 234 of the human GR $\alpha$ was changed to a proline in the mouse, dog, and guinea pig receptors.

In addition to phosphorylation, GR $\alpha$ undergoes other types of posttranslational modifications, such as acetylation,
(A)

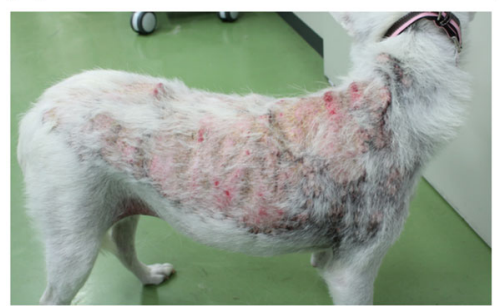

(B)

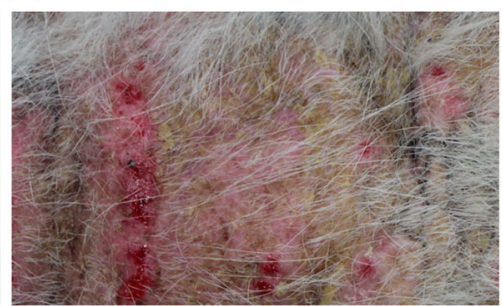

Fig. 1 Bilateral hair loss on truncal regions and calcinosis in a dog with iatrogenic Cushing syndrome. a Alopecia and calcinosis cutis on the dorsal region of this dog. $\mathbf{b}$ Close-up image of lesions exhibiting calcinosis cutis in (a) 
Table 1 Hematological and biochemical findings in the dog on day 1

\begin{tabular}{|c|c|c|c|}
\hline Red blood cells & $853 \times 10^{4} / \mu l$ & BUN & $10.6 \mathrm{mg} / \mathrm{dl}$ \\
\hline Hemoglobin & $19.5 \mathrm{~g} / \mathrm{dl}$ & Creatinine & $0.6 \mathrm{mg} / \mathrm{dl}$ \\
\hline Hematocrit & $53 \%$ & AST & $24 I U / I$ \\
\hline Mean corpuscular volume & $64.0 \mathrm{fl}$ & ALT & $52 \mathrm{IU} / \mathrm{I}$ \\
\hline Mean corpuscular hemoglobin & $22.9 \mathrm{pg}$ & ALP & $511 \mathrm{IU} / \mathrm{I}$ \\
\hline \multirow[t]{2}{*}{ Mean corpuscular hemoglobin concentration } & $35.7 \mathrm{~g} / \mathrm{dl}$ & GGT & $10 \mathrm{IU} / \mathrm{I}$ \\
\hline & & Albumin & $3.0 \mathrm{~g} / \mathrm{dl}$ \\
\hline White blood cells & $7329 / \mu l$ & Total Cholesterol & $197 \mathrm{mg} / \mathrm{dl}$ \\
\hline Band neutrophils & $73 / \mu l$ & Glucose & 99 mg/dl \\
\hline Segmented neutrophils & $5864 / \mu l$ & $\mathrm{Na}$ & $148 \mathrm{mEq} / \mathrm{l}$ \\
\hline Lymphocytes & $586 / \mu l$ & $\mathrm{~N}$ & $3.8 \mathrm{mEq} / \mathrm{l}$ \\
\hline Monocytes & $220 / \mu l$ & $\mathrm{Cl}$ & $114 \mathrm{mEq} / \mathrm{l}$ \\
\hline \multirow[t]{2}{*}{ Eosinophil } & $586 / \mu \mathrm{l}$ & $\mathrm{Ca}$ & $9.5 \mathrm{mg} / \mathrm{dl}$ \\
\hline & & $\mathbb{I P}$ & 4.4 mg/dl \\
\hline Platelet & $44.0 \times 10^{4} / \mu \mathrm{l}$ & CRP & $0.3 \mathrm{mg} / \mathrm{dl}$ \\
\hline Total protein & $7.0 \mathrm{~g} / \mathrm{dl}$ & & \\
\hline
\end{tabular}

SUMOylation, and ubiquitination (Fig. 3) [10-12]. All reported modification sites in the human GR $\alpha$ were completely conserved in the mouse, dog, and guinea pig receptors.

A number of inactivating mutations (N72D, V321A, V423A, R477H, I559D, V571A, D641V, G679S, V729I, F737S, I747M, and L773P) in the human GR $\alpha$ gene are known to cause familial GC resistance [1-3] (Fig. 3). Compared with the inactivating mutations reported in the human GR $\alpha$, most of sites were well conserved between the mouse, dog, and guinea pig receptor genes. However, the dog GR has a histidine at residue 72 rather than an asparagine.

\section{Nucleotide and deduced amino acid sequences of the GR CDNA of the treated dog}

PCR amplification of the cDNA of the dog treated at our hospital using the primers YTM673 and YTM674 generated a DNA fragment with a slightly larger size than an expected size. The nucleotide amino acid sequence of this $G R$ clone was aligned with that of dog wild type GR (Fig. 2), which revealed that the $G R$ of the dog undergoing treatment had an extra 69 nucleotides between nucleotides 2032 and 2033 compared with dog wild type GR. These extra 69 nucleotides matched a part of the nucleotide sequence of dog genomic DNA corresponding to intron 6 (Fig. 4a). Insertion of these extra 69 nucleotides between exons 6 and 7 introduced a frameshift and a premature termination codon (TGA) $15 \mathrm{bp}$ downstream of the insertion. This insertion is thus predicted to result in a truncated protein of 682 amino acids, compared to the normal (wild type) 780 amino acids (Fig. 4b).

\section{Overexpression of dog GR in COS-7 cells}

To confirm the molecular size of the treated dog's GR (cGR $\triangle \mathrm{LBD}$ ) cloned in this study, FLAG-tagged cGR $\triangle \mathrm{LBD}$ or wild type GR were transfected into COS-7 cells, and the expression of the GR was examined by western blotting using an anti-FLAG antibody (Fig. 5a). A single band of the expected molecular weight and several bands of lower molecular weight were observed in COS-7 cells transfected with FLAG-tagged dog GR in COS-7 cells, whereas a band showing a lower molecular weight than wild type GR was confirmed in COS-7 cells transfected with the FLAG-tagged cGR $\triangle \mathrm{LBD}$.

\section{Sensitivity of the treated dog's GR to prednisolone}

To compare the sensitivity to glucocorticoids of the treated dog GR and wild type GR, the luciferase activity of the glucocorticoid response element (GRE) of mouse mammary tumor virus (MMTV) was determined in COS-7 cells transfected with either $G R \alpha$ expression vector, followed by treatment with various concentrations of prednisolone (Fig. 5b and c). The concentration of prednisolone resulting in the maximum luciferase activity was $2.0 \times 10^{-3} \mu \mathrm{M}$ for the normal, healthy dog (Fig. 5b). The calculated $\mathrm{EC}_{50}$ of dog GR-induced luciferase activity was $0.2 \times 10^{-4} \mu \mathrm{M}$.

To determine the effect of deletion of the LBD in cGR $\triangle$ LBD on glucocorticoid sensitivity, the luciferase activity of the GRE was determined in COS-7 cells transfected with cGR $\triangle \mathrm{LBD}$ and treated with various concentrations of prednisolone. The luciferase activity of cGR $\triangle \mathrm{LBD}$ was very low, irrespective of the concentration of glucocorticoid, and unaltered by any increase in the prednisolone concentration $\left(10^{-4}\right.$ to $\left.10^{-2} \mu \mathrm{M}\right)$. However, the activity increased slightly 


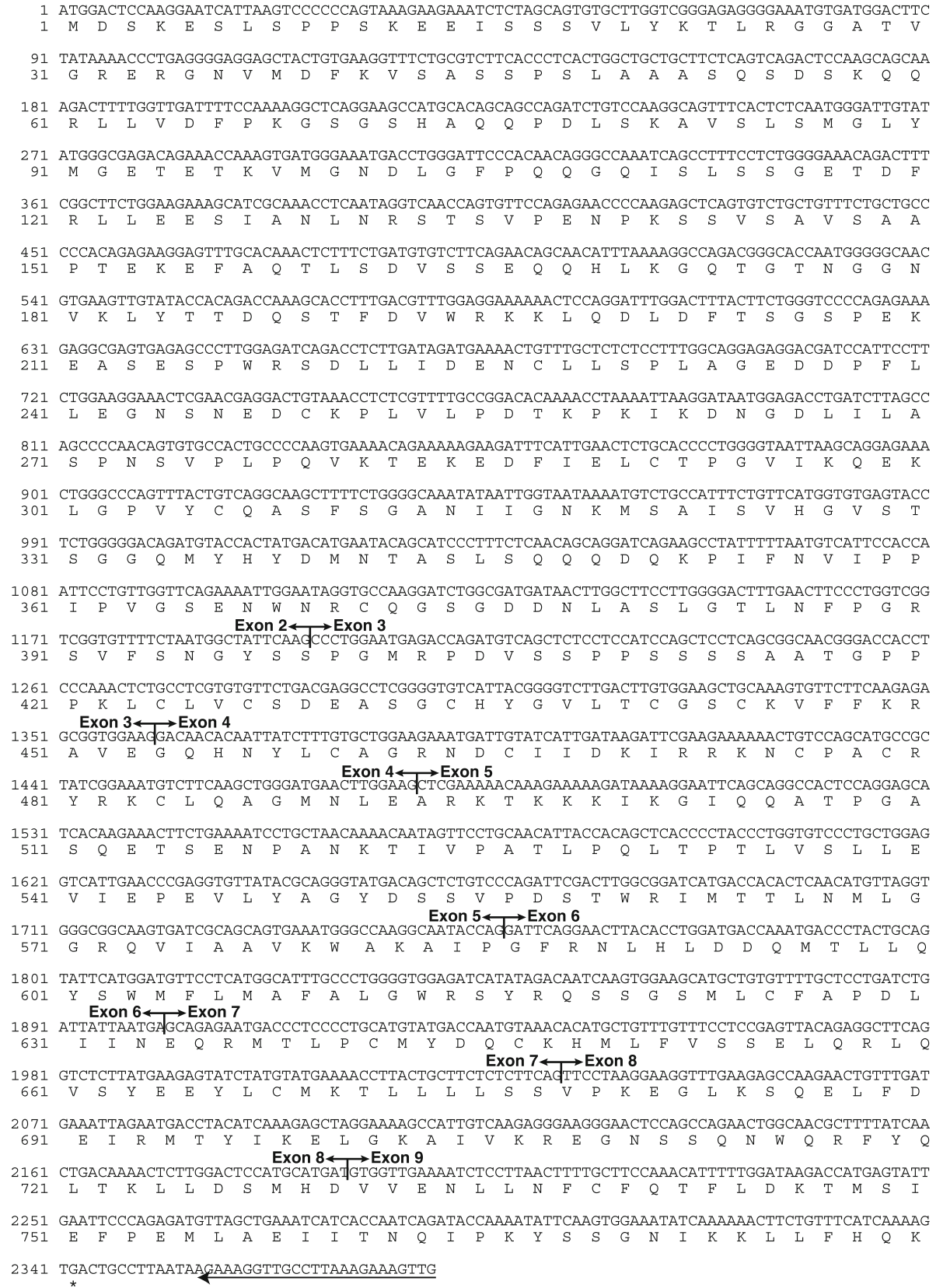

Fig. 2 Nucleotide sequence and predicted amino acid sequence of dog GR CDNA. The nucleotide sequence of dog GR is shown, with the predicted amino acid sequence shown below the nucleotide sequence. Numbers to the left refer to the nucleotide position in the dog GR cDNA or amino acid position in the dog GR. Arrows indicate the primers used for cDNA cloning. The asterisk indicates the stop codon. The nucleotide sequence data reported in this paper were submitted to the DNA Data Bank of Japan (DDBL)/European Molecular Biology Laboratory (EMBL)/ GenBank (Accession No. AB874470). Vertical lines indicate exon-exon boundaries

at very high prednisolone concentrations $\left(10^{-1}\right.$ to $\left.1 \mu \mathrm{M}\right)$ (Fig. 5c).

\section{Discussion}

In this study, we first isolated the dog $G R$ gene from a healthy normal dog and compared its glucocorticoid sensitivities to that of the $G R$ of a dog being treated at our hospital. The dog was being treated for suspected iatrogenic Cushing syndrome, and its GR was characterized by a deletion, which was designated cGR $\triangle \mathrm{LBD}$. We were not able to obtain a complete history regarding the clinical symptoms and medications for this dog, but 


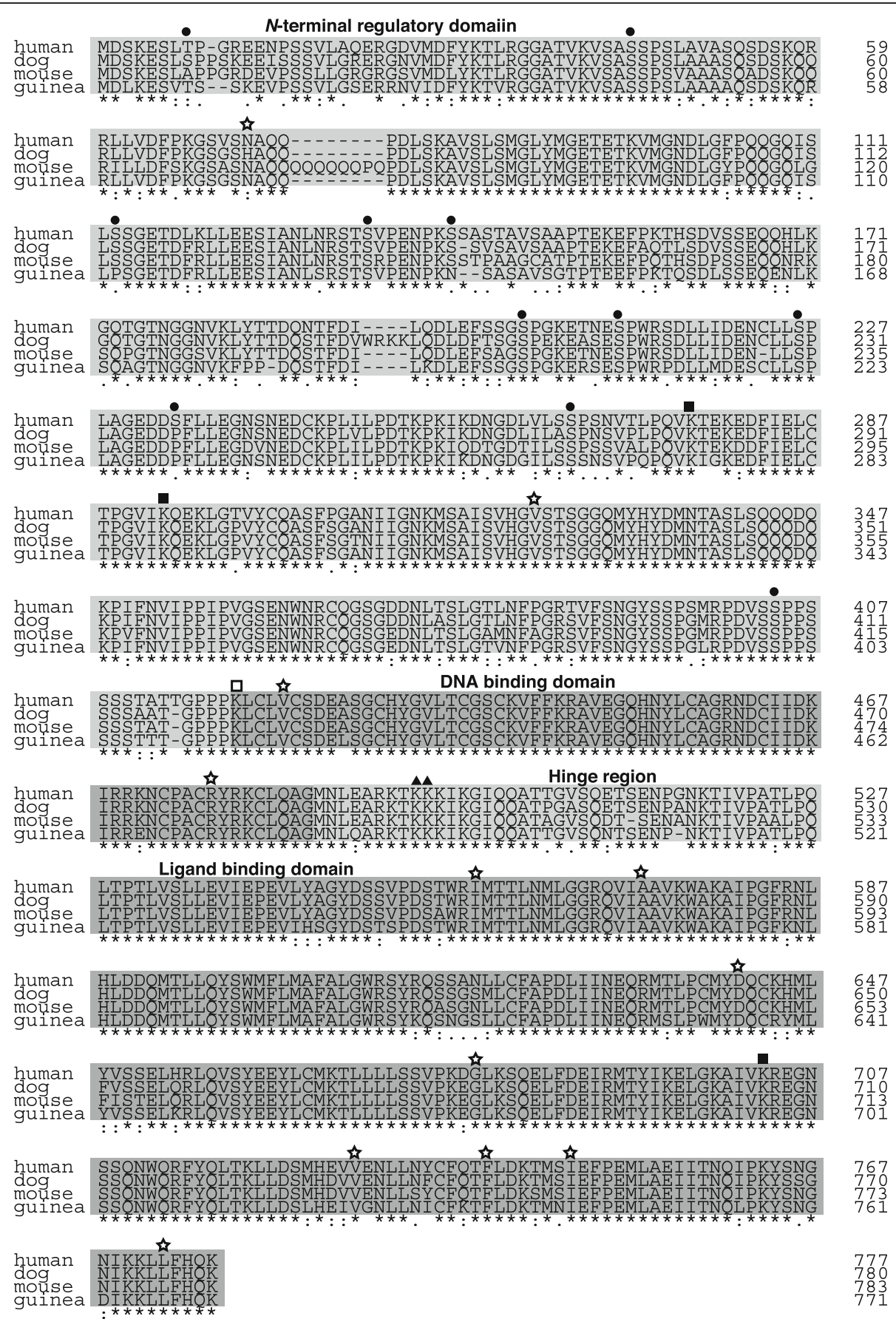

Fig. 3 Comparison of the predicted amino acid sequences of GRs from different species. The amino acid sequence of the dog GR was aligned with that of human, mouse, and guinea pig GRs using Clustal W software. The "*" below columns in the amino acid sequences indicate that the residues in that column are identical in all sequences in the alignment. The ":" and "." symbols indicate that semiconserved substitutions were observed. Numbers to the right refer to amino acid positions. GR amino acid sequences were obtained from the NCBI database (NP_000167.1 for human, P06537.1 for mouse, and NP_01166458.1 for guinea pig). The GR is composed of four domains, which are shown in shaded boxes: Nterminal regulatory domain, DNA-binding domain, hinge region, and ligand-binding domain. Each domain was defined according to the domain composition of the human GR. Closed circles, closed boxes, open box, and closed triangles indicate phosphorylation sites, SUMOylation sites, ubiquitination site, and acetylation sites, respectively, which were identified in the human GR. Stars indicate known mutation sites in the human GR associated with natural resistance 
(A)

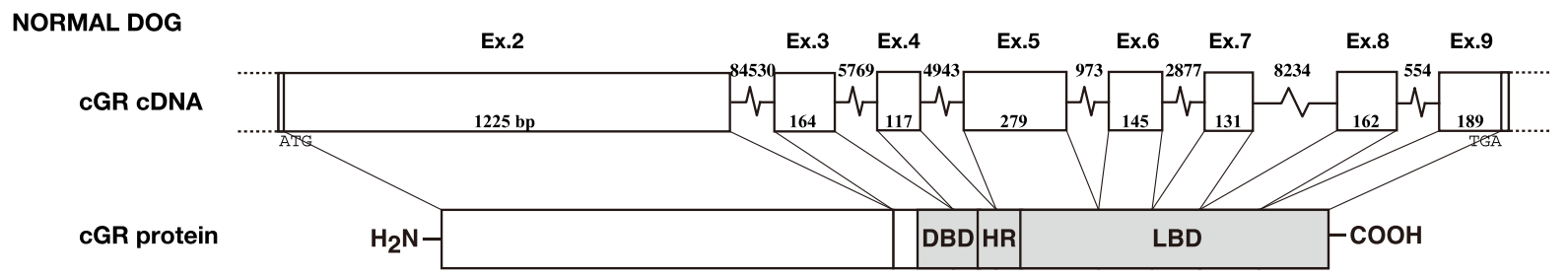

PATIENT DOG

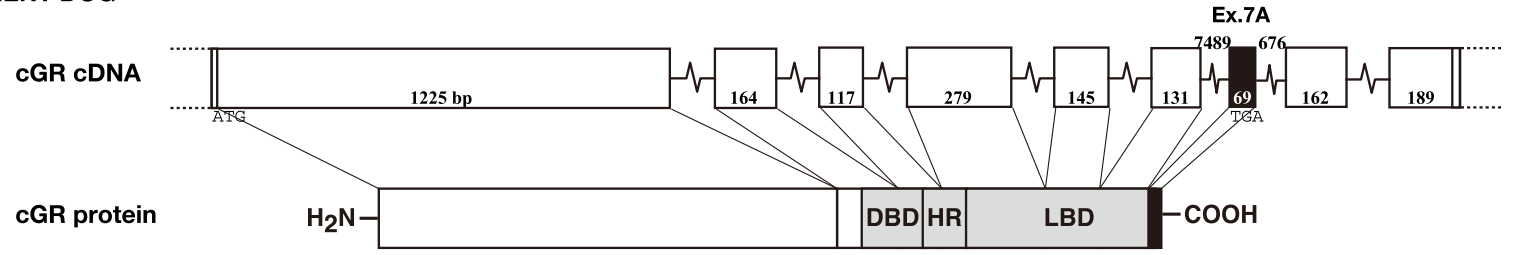

(B)

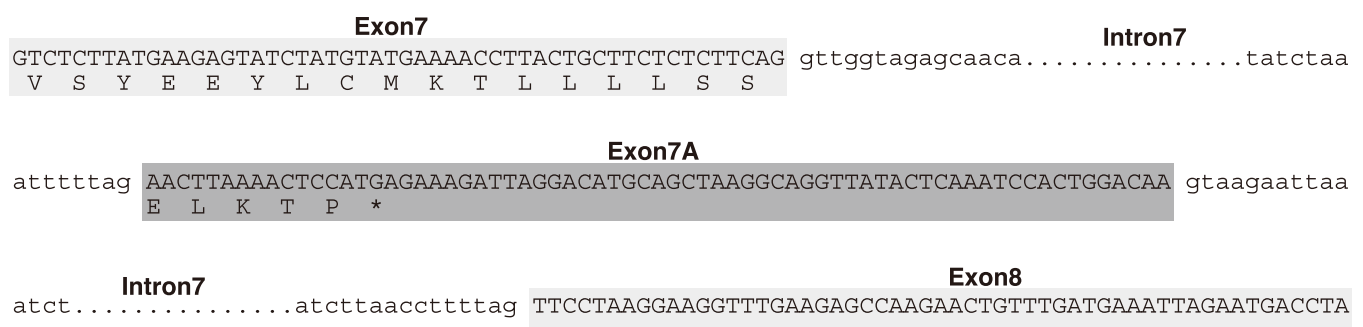

Fig. 4 Structure of the GR gene in the dog with suspected iatrogenic Cushing syndrome. a Each box indicates an individual exon. Exon lengths are show in each box and intron lengths are indicated between each box. The extra exon present only in this dog is depicted in black. $\mathbf{b}$ Partial nucleotide sequence and predicted amino acid sequence of the cDNA of the dog treated in this case. The nucleotide sequence of the GR gene is shown, with the predicted amino acid sequence shown below the nucleotide sequence. The asterisk indicates the stop codon

treatment with prednisolone had not been for an extended period, and the dosage did not seem too high based on the limited information available to us. We then considered that this dog could be very sensitive to prednisolone and hypothesized that the abnormal GR was more sensitive to glucocorticoid than normal dog GR. cGR $\triangle$ LBD had a shorter LBD than the normal dog LBD due to alternative splicing generated by insertion of an extra exon. Furthermore, cGR $\triangle$ LBD exhibited poor sensitivity to prednisolone, in complete contradiction to our hypothesis. On the one hand, this minimal sensitivity of cGR $\triangle \mathrm{LBD}$ to glucocorticoid was consistent with the results of a previous study showing that an artificial LBD-deletion mutant human GR had no sensitivity to glucocorticoid [13]. The LBD plays a role in the binding of glucocorticoid to the GR, and its sensitivity varies. For example, the guinea pig GR has lower affinity for dexamethasone than the mouse and human GRs [14] due to 4 important differences in the amino acid sequence between residues $544-551$ of the LBD. Asparagine 619, leucine 620, tyrosine 735, and asparagine 766 in the LBD of hGR are conserved only in the human and mouse GR, but in the $\operatorname{dog}$ GR, these residues are substituted with serine, methionine, phenylalanine, and serine, respectively. These differences could contribute the increased sensitivity of dog GR to prednisolone as compared with the human and mouse GRs.

In humans, no or little sensitivity to glucocorticoid is known as cortisol resistance and typically results from a mutation in the LBD [1]. Among these mutations, I559N and $I 747 \mathrm{M}$ are known as dominant negative mutations [15], which lead to clinical syndromes such as cortisol resistance. Patients with these mutations present with increased cortisol levels compared with the normal population. The negative glucocorticoid feedback controlling secretion of both corticotrophin-releasing hormone (CRH) and adrenocorticotrophin (ACTH) is suppressed as a consequence of diminished glucocorticoid sensitivity. Secretion of both $\mathrm{CRH}$ and $\mathrm{ACTH}$ increases, resulting in higher serum cortisol levels, which appear to compensate adequately for the reduced sensitivity. As the increased serum cortisol levels are physiologically needed, patients do not 
(A)

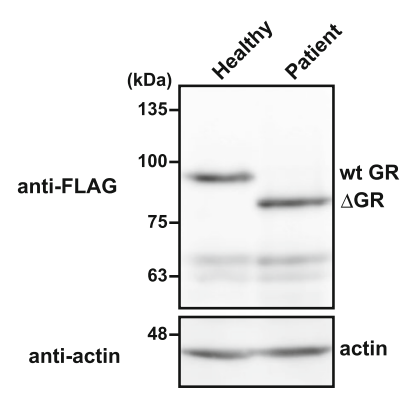

(B)

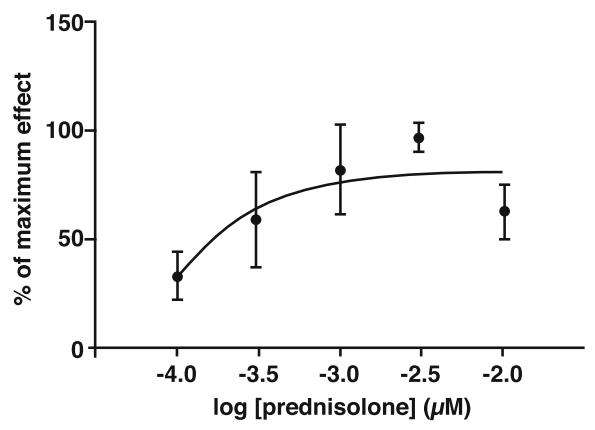

(C)

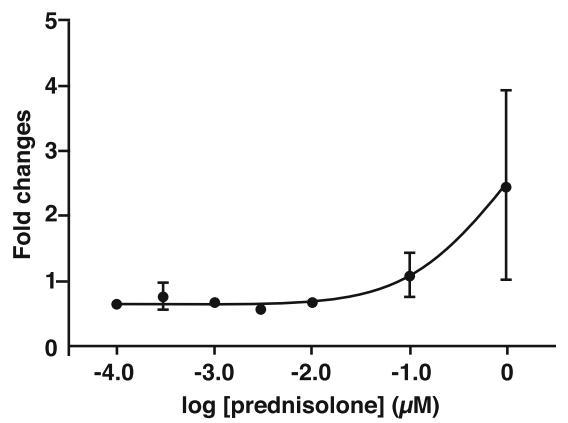

Fig. 5 Expression and sensitivity of $C G R \Delta L B D$ to prednisolone. a COS-7 cells were transiently transfected with pCR3-cGR\#3 or a cGR LLBDexpressing vector. Forty-eight hours after transfection, whole cell lysates were prepared and analyzed by western blotting using an anti-FLAG antibody (M2). The results of 1 of 2 comparable experiments are shown. $\mathbf{b}, \mathbf{c}$ Lower responsiveness of CGR LLBD to prednisolone. COS-7 cells were transiently transfected with pGL4.36, pRL-SV40, and either a cGR- or CGR LLBD-expressing vector and then treated with different doses of prednisolone $\left(10^{-4}\right.$ to $\left.1 \mu \mathrm{M}\right)$ for $24 \mathrm{~h}$. Transactivation of cGR (b) or CGRALBD (c) was evaluated using a luciferase assay. Values were calculated as $\%$ of maximal effect (b) or fold-increase in reactivity to basal treatment (c) and represent the means \pm SE of more than three independent experiments

show symptoms of cortisol excess [16]. The diagnostic criteria for generalized glucocorticoid resistance include no clinical signs of Cushing syndrome, increased plasma total and free cortisol (normal plasma cortisol-binding globulin concentration), 24-h urinary free cortisol or 17-hydroxysteroid excretion, increased rate of cortisol production, and resistance to dexamethasone [17]. As we did not perform all of these examinations, we cannot completely exclude the possibility that this dog had cortisol resistance. However, it is unlikely that this dog had generalized cortisol resistance because it did not exhibit increased plasma cortisol (baseline cortisol of $1.5 \mu \mathrm{g} / \mathrm{dL}$ and $6.18 \mu \mathrm{g} / \mathrm{dL}$ at 3 weeks and 17 months after the first visit to our hospital, respectively) but did exhibit increased alkaline phosphatase after treatment with prednisolone at the private veterinary hospital.

The discrepancy between this dog's clinical response to glucocorticoid and harboring a glucocorticoid-unresponsive form of GR could be explained as follows. Whereas human patients exhibiting generalized glucocorticoid resistance have genomic mutations in the LBD of the GR, the treated dog of this case harbored an alternative splicing variant $G R$. This could occur only in peripheral blood mononuclear cells (PBMCs) from this dog; if other tissues had GRs with a normal splicing pattern, generalized glucocorticoid resistance should not be observed. Unfortunately, we were not able to obtain other tissues from this dog and thus could not determine the expression pattern of GRs in other tissues. Moreover, we were not able to obtain genomic DNA from this dog, which could have provided information as to whether this mutant form of $G R$ derived from germ-line mutations that could induce the abnormal splicing pattern. In this study, we also cloned the GR from the PBMCs from two normal healthy dogs, as well as the thymus of a normal, healthy dog. On the other hands, this dog had only mutant form of GR in PBMC. This suggested that the mutant form of GR, cGR $\triangle \mathrm{LBD}$, is characteristics of $G R$ in PBMC from this dog.

In this study, we conducted a molecular characterization of the dog GR. We also identified a natural mutant GR in a dog being treated for suspected iatrogenic Cushing syndrome and compared its function with that of the GR of a normal, healthy dog. The precise mechanism controlling the sensitivity of the GR in dogs remains to be elucidated, but the results of this study could facilitate further studies of dog GR sensitivity and/or resistance. 


\section{Conclusions}

In this study, we molecular cloned canine $G R$ for the first time. We also identified a mutated $G R$ from the dog with iatrogenic cushing's syndrome. This mutated $G R$ had very low sensitivity to glucocorticoid treatment as shown by GR-responsive reporter gene assay. Although the significance of this mutated $G R$ in iatrogenic cushing's syndrome is still unknown, but this is the first report about abrogated canine GR from patient dog.

\section{Methods}

\section{Cell culture}

COS-7 (African Green monkey kidney) cells were grown in R10 complete medium (RPMI 1640; Nacalai Tesque, Kyoto, Japan) supplemented with $10 \%$ fetal calf serum, $55 \mu \mathrm{M}$ 2-mercaptoethanol, $100 \mathrm{units} / \mathrm{ml}$ penicillin, and $100 \mu \mathrm{g} / \mathrm{ml}$ streptomycin. Cells were incubated at $37^{\circ} \mathrm{C}$ in a humidified atmosphere containing $5.0 \% \mathrm{CO}_{2}$.

\section{Molecular cloning of canine GRs}

A whole blood was obtained from a dog undergoing veterinary treatment under the dog's owner consent, and was used to clone the full-length dog GR. One microgram of total RNA was isolated from each specimen, treated with Turbo DNA-free (Ambion Life Technologies, CA), and then transcribed into cDNA using Superscript III (Invitrogen Life Technologies, CA), according to the manufacturer's instructions. Oligo dT primers were used to prime the first-strand synthesis for each reaction. For a control dog sample, the frozen stocked cDNA of canine thymus and PBMCs in previous study \{Mizuno:2009tw\} was used.

The primers to amplify dog GR cDNA, YTM673 (5'TTTTGCCTGGTAGCTGATGA-3') and YTM674 (5'CAACTTTCTTTAAGGCAACCTTTC-3'), were designed based on predicted sequences of the $G R$ as determined from the dog genomic database (GenBank Accession No. NW_876266.1). Using these primers, the dog GR gene was amplified from cDNA using a KOD plus kit (Toyobo, Osaka, Japan) according to the manufacturer's instructions. Pre-denaturation at $94^{\circ} \mathrm{C}$ for $2 \mathrm{~min}$ was followed by 35 cycles of PCR amplification consisting of denaturation at $94{ }^{\circ} \mathrm{C}$ for $10 \mathrm{~s}$, annealing at $60{ }^{\circ} \mathrm{C}$ for $30 \mathrm{~s}$, extension at $68^{\circ} \mathrm{C}$ for $3 \mathrm{~min}$, and then a final extension at $68^{\circ} \mathrm{C}$ for $10 \mathrm{~min}$ using a PCR System 9700 (Perkin-Elmer, Waltham, MA). Each gel-purified PCR product was inserted into SmaI restriction sites of the pBluescript SK (-) vector (designated pBS-GR $\alpha$ full\#1). The constructed insert-containing vector was sequenced using M13 (-20) forward primer and M13 reverse primer with a BigDye Termination v3.1 Cycle Sequencing kit (Perkin-Elmer) and analyzed using a PRISM3100-Avant sequencer (Applied Biosystems, Foster City, CA) at the Yamaguchi University Center for Gene Research. The resulting sequencing data were compared to the previously reported GR using ApE (A plasmid Editor) and Clustal W software.

\section{Construction of expression vectors}

To obtain the vector encoding the $\operatorname{dog} G R$, two FLAG-tag sequences were added at the N-terminus of the GR. PCR was carried out with each primer pair, YTM1033 (5'-AGGAATTCGACTCCAAGGAATCATA-3') and YTM1034 (5'-CCAGAGGAAAGGCT GATTTG-3'), using pBS-GR $\alpha$ full\#1 as a template. The PCR product was purified and digested with $B g l$ and EcoRI. To obtain the second half of the cGR, pBS-GR $\alpha$ full\# 1 was digested with $B g l \mathrm{I}$ and $\mathrm{XbaI}$. The amplified product carrying the FLAG tag and the corresponding second half digested with restriction enzymes were ligated into the EcoRI and XbaI sites of the pCR2xFL vector. The nucleotide sequence of the clone was confirmed by sequencing, as described above.

\section{Western blot analysis}

For transfection, cells were plated in a 6-well plate at $3.0 \times 10^{5}$ cells in $2 \mathrm{ml}$ of $\mathrm{R} 10$ medium $24 \mathrm{~h}$ prior to transfection. Cells were transfected using TransIT-LT1 (Mirus Bio Corporation, WI) transfection reagent according to the manufacturer's instructions. In brief, $1 \mu \mathrm{l}$ of TransIT-LT1 and $1 \mu \mathrm{g}$ of one of the GR expression vectors were mixed in $100 \mu \mathrm{l}$ of un-supplemented RPMI 1640 medium. After incubation for $15 \mathrm{~min}$ at room temperature (RT), the mixture was added to the cells. At $48 \mathrm{~h}$ after transfection, the cells were washed with $\mathrm{D}$ PBS(-) and stored at $-80^{\circ} \mathrm{C}$ until use.

For western blot analysis, cells were pelleted and then lysed with $1 \%$ NP40 lysis buffer $(150 \mathrm{mM} \mathrm{NaCl}, 10 \mathrm{mM}$ Tris- $\mathrm{HCl}$ [pH 7.5], $1 \mathrm{mM}$ EDTA, $1 \mathrm{mM} \mathrm{PMSF,} \mathrm{and}$ complete Mini EDTA-free protease inhibitor mixture [Roche Diagnostics K.K., Tokyo, Japan]), centrifuged at $15,000 \mathrm{rpm}$ for $15 \mathrm{~min}$ at $4{ }^{\circ} \mathrm{C}$, and then the supernatant was collected. Extracted proteins were separated by $8 \%$ SDS-PAGE and then transferred onto a PVDF membrane (Hybond-P GE Healthcare, Little Chalfont, U.K.). The membrane was blocked with $5 \%$ nonfat dry milk in $1 \times$ TBS-T ( $1 \times$ TBS, $0.05 \%$ Tween-20) for $1 \mathrm{~h}$ at RT and then incubated with anti-FLAG antibody (clone M2, 1: 1000; Sigma-Aldrich, St. Louis, MO) overnight at $4{ }^{\circ} \mathrm{C}$. The membrane was then washed three times with TBS-T for $10 \mathrm{~min}$ and incubated with horseradish peroxidaseconjugated goat anti-mouse IgG polyclonal secondary antibody (Zymed, South San Francisco, CA) (1:4000 dilution) for $1 \mathrm{~h}$ at RT. The membrane was washed three times with TBS-T for $10 \mathrm{~min}$ each and visualized by immersion in Western Lightning Chemiluminescence reagent (Perkin-Elmer). Immunoreactive bands were quantified using a Las 3000 mini luminescent image analyzer (Fujifilm, Tokyo, Japan), and the data were analyzed using 
Science Lab 2005 (Fujifilm). Immunoblots were then stripped and re-probed with anti-actin antibody (clone C11, 1:1000; Santa Cruz Biotechnology, Inc).

\section{Luciferase assay}

At $24 \mathrm{~h}$ prior to transfection, cells were plated at $2.0 \times 10^{5}$ cells in $1 \mathrm{ml}$ of R10 medium in a 12-well plate. Cells were transfected using TransIT-LT1 (Mirus Bio Corp.) transfection reagent. In brief, $3 \mu \mathrm{l}$ of TransIT-LT1, $330 \mathrm{ng}$ of pGL4.36 (Promega, Madison, WI), 10 ng of pRL-SV40 (Promega), and $670 \mathrm{ng}$ of one of the GR-expressing vectors in $100 \mu \mathrm{l}$ of Opti-MEM serum-free medium (Gibco ${ }^{\circ}$, Invitrogen Life Technologies, CA) were mixed. Twenty-four hours after transfection, cells were detached and re-plated in a 96-well plate $\left(2.0 \times 10^{4}\right.$ cells in $75 \mu \mathrm{l}$ of R10 medium) in triplicate. Cells were incubated for an additional $24 \mathrm{~h}$, and then serial dilutions of prednisolone were added to each well. After $24 \mathrm{~h}$ of incubation, the cells were lysed using a Dual-Glo ${ }^{\mathrm{Tm}}$ Luciferase Assay System (Promega), and luciferase activity was assessed using a DTX800 Multimode Detector (Beckman Coulter, Brea, CA) according to the manufacturer's instructions. Firefly luciferase activity was normalized to renilla luciferase activity generated from the pRL-SV40 vector in the same cells. $E_{50}$ values were calculated based on concentration-response curves using GraphPad Prism6 software (GraphPad, Inc., La Jolla, CA).

\section{Abbreviations}

ACTH: Adrenocorticotrophin; CRH: Corticotrophin-releasing hormone; DBD: DNA-binding domain; GR: Glucocorticoid receptor; GRE: Glucocorticoid response element; LBD: Ligand-binding domain; MMTV: Mouse mammary tumor virus; NTD: N-terminal regulatory domain; PBMCs: Peripheral blood mononuclear cells; SD: Standard deviation

\section{Acknowledgments}

We acknowledge the technical expertise of the DNA Core Facility of the Center for Gene Research, Yamaguchi University.

\section{Authors' contributions}

$\mathrm{KY}$ and TM were involved in case analysis and was responsible for writing the manuscript. MO was responsible for interpretation of results. All authors read and approved the final manuscript.

\section{Funding}

Not applicable

\section{Availability of data and materials}

The datasets used and/or analyzed during the current study are available from the corresponding author on reasonable request.

\section{Ethics approval and consent to participate}

This study was approved by the ethics committee of Yamaguchi University Animal Medical Center. Informed consent for taking blood was verbally obtained from the owner and was filled in medical chart. The committee approved the verbal consent at that stage because the comprehensive prior consent arrangement was also taken with documents at the first visit.

\section{Consent for publication}

We obtained a written consent from the owner to use a dog's picture.

\section{Author details}

'Laboratory of Molecular Diagnostics and Therapeutics, Joint Faculty of Veterinary Medicine, Yamaguchi University, 1677-1 Yoshida, Yamaguchi-shi, Yamaguchi 753-8515, Japan. ${ }^{2}$ Laboratory of Veterinary Internal Medicine, Joint Faculty of Veterinary Medicine, Yamaguchi University, 1677-1 Yoshida, Yamaguchi-shi, Yamaguchi 753-8515, Japan.

Received: 15 April 2019 Accepted: 2 October 2019

Published online: 24 October 2019

\section{References}

1. Charmandari E, Kino T, Ichijo T, Chrousos GP. Generalized glucocorticoid resistance: clinical aspects, molecular mechanisms, and implications of a rare genetic disorder. J Clin Endocrinol Metab Endocrine Soc. 2008;93:1563-72.

2. Baker AC, Chew WW, Green TL, Tung K, Lim D, Cho K, et al. Single nucleotide polymorphisms and type of steroid impact the functional response of the human glucocorticoid receptor. J Surg Res. 2013;180:27-34.

3. Roberts ML, Kino T, Nicolaides NC, Hurt DE, Katsantoni E, Sertedaki A, et al. A novel point mutation in the DNA-binding domain (DBD) of the human glucocorticoid receptor causes primary generalized glucocorticoid resistance by disrupting the hydrophobic structure of its DBD. J Clin Endocrinol Metab. 2013;98:E790-5.

4. Keightley MC, Fuller PJ. Unique sequences in the Guinea pig glucocorticoid receptor induce constitutive transactivation and decrease steroid sensitivity. Mol Endocrinol. 1994;8:431-9.

5. Trebble P, Matthews L, Blaikley J, Wayte AWO, Black GCM, Wilton A, et al. Familial glucocorticoid resistance caused by a novel Frameshift glucocorticoid receptor mutation. J Clin Endocrinol Metab. 2010;95:E490-9.

6. Lowe AD, Campbell KL, Graves T. Glucocorticoids in the cat. Vet Dermatol. 2008:19:340-7.

7. Yang N, Ray DW, Matthews LC. Current concepts in glucocorticoid resistance. Steroids. 2012;77:1041-9.

8. Krstic MD, Rogatsky I, Yamamoto KR, Garabedian MJ. Mitogen-activated and cyclin-dependent protein kinases selectively and differentially modulate transcriptional enhancement by the glucocorticoid receptor. Mol Cell Biol. 1997;17:3947-54.

9. Dephoure N, Zhou C, Villén J, Beausoleil SA, Bakalarski CE, Elledge SJ, et al. A quantitative atlas of mitotic phosphorylation. Proc Natl Acad Sci U S A. 2008;105:10762-7.

10. Garside H, Waters C, Berry A, Rice L, Ardley HC, White A, et al. UbcH7 interacts with the glucocorticoid receptor and mediates receptor autoregulation. J Endocrinol. 2006;190:621-9.

11. Beck IME, Vanden Berghe W, Vermeulen L, Yamamoto KR, Haegeman G, De Bosscher K. Crosstalk in inflammation: the interplay of glucocorticoid receptor-based mechanisms and kinases and phosphatases. Endocr Rev. 2009:30:830-82

12. Ito K, Yamamura S, Essilfie-Quaye S, Cosio B, Ito M, Barnes PJ, et al. Histone deacetylase 2-mediated deacetylation of the glucocorticoid receptor enables NF-kappaB suppression. J Exp Med. 2006;203:7-13.

13. Hollenberg SM, Giguere V, Evans RM. Identification of two regions of the human glucocorticoid receptor hormone binding domain that block activation. Cancer Res. 1989:49:2292s-4s.

14. Keightley MC, Fuller PJ. Cortisol resistance and the Guinea pig glucocorticoid receptor. Steroids. 1995;60:87-92.

15. Kino T, Vottero A, Charmandari E, Chrousos GP. Familial/sporadic glucocorticoid resistance syndrome and hypertension. Ann N Y Acad Sci. 2002;970:101-11.

16. Lamberts SWJ. Glucocorticoid receptors and Cushing's disease. Mol Cell Endocrinol. 2002;197:69-72.

17. Arai K, Chrousos GP. Hormone-nuclear receptor interactions in health and disease. Glucocorticoid resistance. Baillieres Clin Endocrinol Metab. 1994:8:317-31.

\section{Publisher's Note}

Springer Nature remains neutral with regard to jurisdictional claims in published maps and institutional affiliations. 